\title{
THE CLINICAL APPLICATION OF THE ELECTROENCEPHALOGRAM IN CEREBRAL TUMOUR
}

\author{
By Gerald Parsons-Smith, M.D., M.R.C.P. \\ Physician, West End Hospital for Nervous Disease; Neurologist, Graylingwell Hospital; \\ Consultant in Electroencephalography, West London Hospital
}

The introduction of electroencephalographic study to cases of cerebral tumour has provided the neurologist, during the past 15 years, with a new dimension in diagnostic approach which he can inter-relate with the other methods of investigation at his disposal. In 1931 Berger showed that impairment of cortical function resulting from high intracranial pressure was associated with slow frequency potentials. Two years later he found similar waves confined to the affected hemispheres in two cases of cerebral tumour (Berger, 1933). No particular importance was attached to these slow waves until Walter (1936) found a definite focus of origin of these abnormal frequencies at a point on the skull under which, at autopsy at a later date, a tumour was discovered to be involving the cerebral cortex. Adrian (1935) had already forecast that the study of the electroencephalogram might be of use to the clinician, and it was as a result of this discovery by Walter that interest in the clinical uses of the electroencephalogram (E.E.G.) was aroused.

The clinician can now obtain in the E.E.G. a permanent visual record of the distribution of the abnormal cortical waves, together with those normal ones which may persist, and he can use it to provide an objective physiological background to the complex clinical picture which may be caused by a cerebral tumour. The usual technique, which causes no discomfort to the patient, consists in placing 12 electrodes over the patient's scalp in a standard arrangement such as is described by Cobb (1950). The recording session generally lasts for one hour, during which time the patient lies quietly on a couch with the eyes shut.

It did, at first, seem probable that the E.E.G. would prove an exceedingly accurate diagnostic technique, as the earlier published results on selected cases were impressive, so that it was considered that if such exact localization of a tumour could be obtained consistently by this relatively simple and safe method, then clearly it must be the method of choice. But in spite of great improvements in equipment, technique and skill in interpretation which have taken place during the past decade, the results in many large series of unselected cases have shown that precise localization cannot be guaranteed. The fundamental source of the difficulty in localization is that the abnormal brain waves which are seen in cases of cerebral tumour arise from the altered brain tissue which is adjacent to the tumour, and that the actual substance of most cerebral tumours is electrically silent. Because of this the position of the electrographic abnormality may not always coincide with the site of the tumour as exposed by the neurosurgeon, with the result that the method may be considered inaccurate by the inexperienced.

The claims for exact localization of accessible cerebral tumours are varied, figures from 47.8 to 88.3 per cent. being reported. Examination of I,242 published cases of accessible cerebral tumours collected from 12 sources shows that accurate localization was achieved in 73 per cent. of the patients (Yeager et al., 1940; Gibbs et al., 1941; Cobb, 1944; Hines et al., 1944; Yeager and Luse, 1945; Baudouin et al., 1946; Hoefer et al., 1946; Bassett and Bagchi, 1947; Buchthal and Busch, 1947; Schlesinger and Strauss, 1947; Parsons-Smith, 1949; Kershman et al., 1949). The largest number of cases in any one series was that published by Hoefer, Schlesinger and Pennes (1946), who analyzed the results from the study of 543 cases of verified brain tumours at the Neuro- 
logical Institute in New York. In 416 hemisphere lesions, localization was obtained in 64 per cent. and lateralization in a further 9.6 per cent. In 247 gliomata the figures were 72 per cent. and 8. I per cent., and in 77 meningiomata 47 per cent. and I7 per cent. respectively. These figures compare closely with the author's in a study of $15^{\circ}$ verified cases (Parsons-Smith, 1949). In this series 100 hemisphere tumours were localized in 66 per cent. and lateralized in a further $2 \mathrm{r}$ per cent., whilst the figures for 40 astrocytomata, grades $I$ and 2 , were 74 per cent. and 14.5 per cent., for 40 astrocytomata, grades 3 and 4 (gliobastoma multiforme), 74 per cent. and 13 per cent., and for 20 meningomata 33 per cent. and 33 per cent. Examination of the figures in the two series quoted above show that an abnormality will be seen in the correct hemisphere in 80 to 88 per cent. of the accessible gliomata, but even so the value of E.E.G. study will be more apparent if it is used to throw light on the behaviour of the cerebral tumour rather than to place its position anatomically.

The optimum method of investigation of a patient suspected of having a cerebral tumour is by a combination of clinical, electroencephalographic and radiological techniques, and in this way precise localization can be expected. Although great improvements in the radiological localization figures have taken place recently, the method is not successful in every case. The commoner sources of failure are insufficient amount of air in the ventricles and in not being able to determine the extent of the lesion or whether it is extra- or intracerebral.

Nevertheless, anatomical localization can be expected by X-ray in 85 to 90 per cent. of all cases. The E.E.G. has proved just as accurate an indicator as radiology in the accessible tumours, but the localization which it gives may not always coincide with the site convenient for surgical approach and it should not, therefore, be used for this purpose. It is more profitable to blend the E.E.G. data with the anatomical evidence derived by $\mathrm{X}$-ray so as to throw light on the nature of the tumour, the direction of its spread, and its effect on the surrounding brain tissue.

\section{Limitations and Discrepancies}

The E.E.G. is a valuable diagnostic method in cases of cerebral tumour provided that the physician and the surgeon both appreciate that apparent fallacies may occur, though it is stressed that an accurate physiological explanation can usually be elicited to explain those so-called fallacies. There are limitations to the E.E.G. method and the most. serious are as follows:
I. Only lesions above the tentorium directly气 affect the normal brain waves.

2. The actual substance of a cerebral tumour has been shown to be electrically inactive, and it is now known that the electrical abnormalities $\overrightarrow{\vec{s}}$ arise in the abnormal cerebral tissues around the tumours.

3. The diagnosis of cerebral tumour cannot bew made from the E.E.G. alone, as no one E.E.G $\mathbb{\widetilde { D }}$ appearance is per se specific for a neoplasm.

4. It is not possible to differentiate the various. tumour types on E.E.G. appearances. This is not unexpected. Scherer (1938) showed that when all $\vec{w}$ areas of any one glioma are sectioned it is ofteno impossible to classify it, as one point may show: relatively benign features whilst another may be highly malignant. Even so, diagnostic E.E.G. claims of this sort continue to appear in thei literature.

5. Parasagittal frontal lesions, even when veryo malignant, may not necessarily disturb the norma ${ }_{0}^{\omega}$ cortical rhythms.

6. Mirror foci, and so-called false localizing $\vec{z}_{\vec{Z}}$ signs, are potential sources of the quite gross dis-⿳亠丷厂 crepancies which occur with greater frequencythan many observers admit. Thus in about goo per cent. of tumours involving the cerebelp $N$ pontine angle a severe focal abnormality may $\bar{B} e_{0}$ seen in the contralateral temporal lobe. The faFes localizing signs and mirror foci seen with posterioro fossa lesions present an interesting physiologicalo problem which is not fully understood. It is尺 possible that the action potentials of brain cells $\vec{\Rightarrow}$ and axons are conducted along such connections $\overline{3}$ as the corpus callosum and fronto-pontine tracts. In other cases the false foci may be caused by? oedema resulting upon brain shift or interferenceo with the blood supply. In the following case the 3 . focus must have been significant from the first but was ignored:

A boy of nine developed a staggering gait and signs of raised intracranial pressure. An E.E.G. showed a one- to two-a-second focus in the left parietal area. Moderate hydrocephalus was seen? in the ventriculogram with signs of a left cerebellar tumour. This was confirmed at operation $\tilde{N}$ when a malignant medulloblastoma was exposed: $\mathrm{N}$ The child was treated with radiotherapy. Ten months later the child developed epilepsy involv- $\omega$ ing the right arm and leg, the fits clearly arising in that area of the cortex which had earlier beerfo involved in the E.E.G. abnormality.

7. It is not possible to localize, or even to lateralize, by electroencephalography, tumours of the posterior fossa (see Fig. $\mathrm{r}$ ).

8. Either very high intracranial pressure, or $\frac{?}{\circ}$ severe changes in consciousness, or both, will pro- $\frac{0}{0}$ 


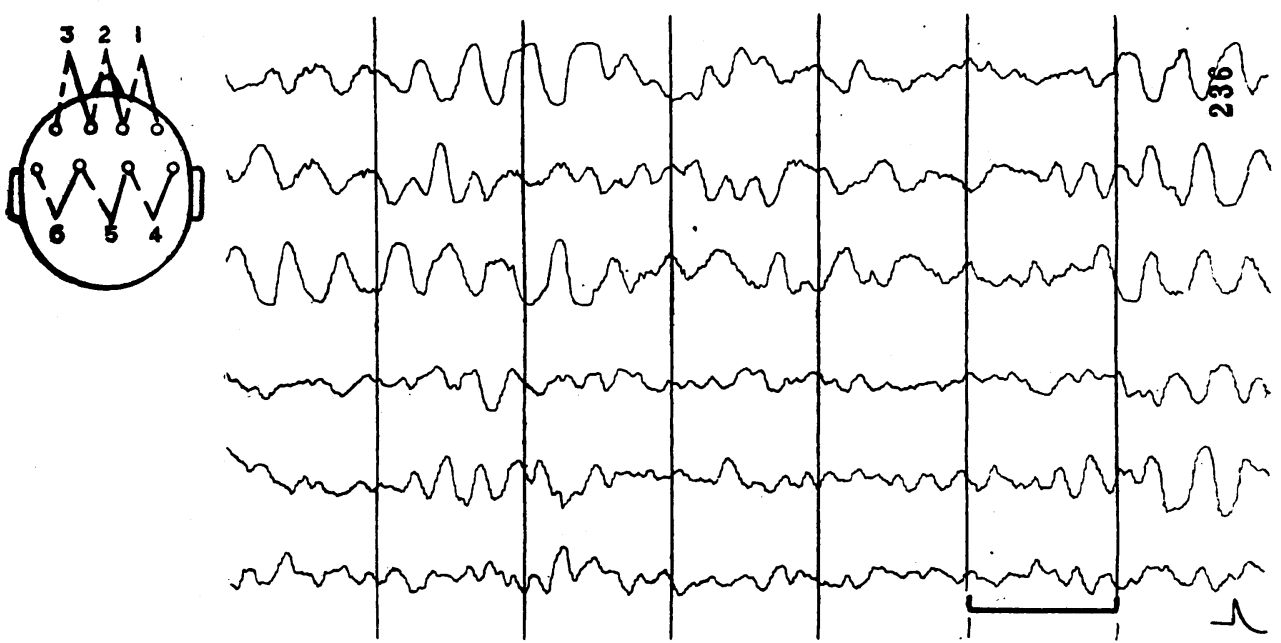

Fig. 1.-Right cerebellar cystic astrocytoma. High voltage three-a-second pressure waves arising from both cerebral hemispheres. First a phase reversal is seen from the left frontal lobe, and later in the record from the right.

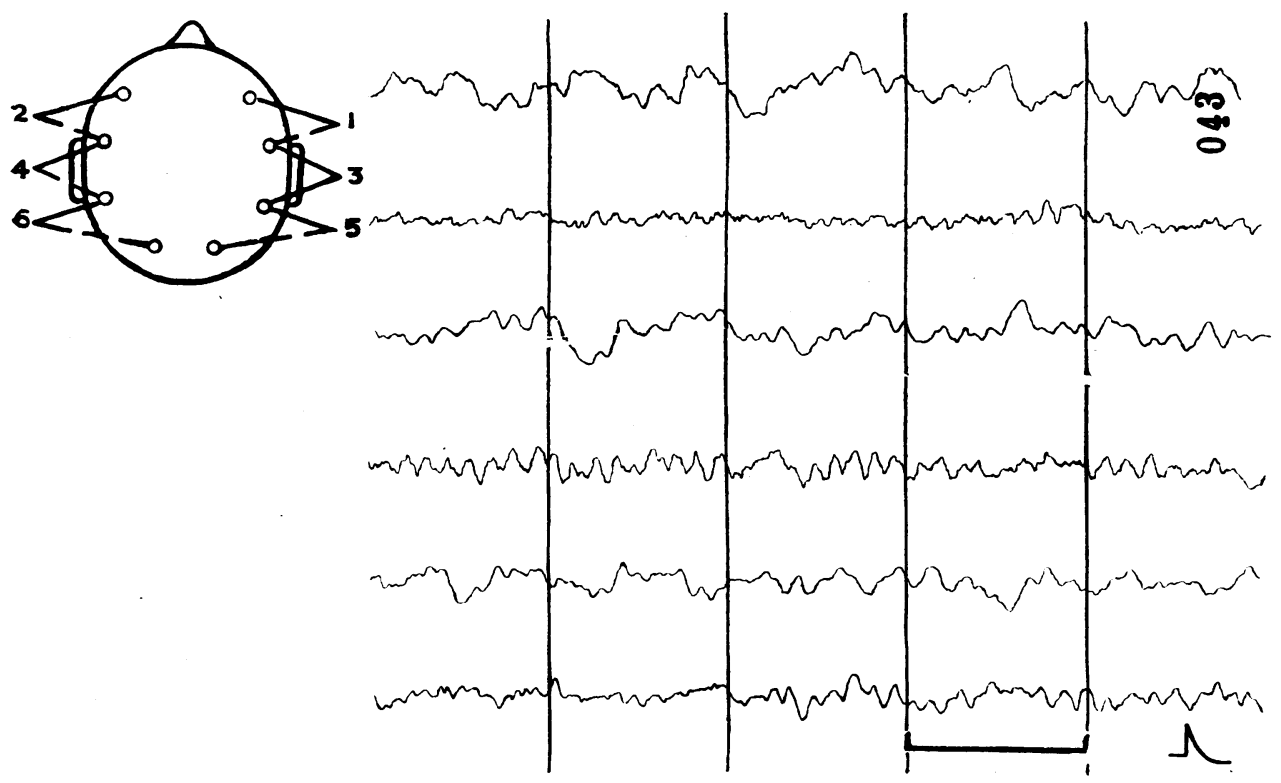

FIG. 2.-Right anterior temporal fibrillary astrocytoma. High voltage one- to two-a-second waves appear in the right anterior temporal lead, with a phase reversal. The posterior temporal lead on the right side contains two- to five-a-second activity. In all the figures the horizontal line represents $I$ second and the vertical signal 50 microvolts. 
duce abnormal waves which may completely mask a severe focal abnormality.

\section{E.E.G. Appearances Produced by Cerebral Tumours}

Various degrees of severity of the E.F.G. abnormality are met with in most cases of cerebral tumour, but in some patients the record may be quite normal. The abnormality is usually a slow wave focus, a delta focus, and its rate may vary from one to seven cycles a second. There is no constant relationship between the rate of the delta focus and the underlying cerebral pathology, though the slower frequencies are more frequently associated with a raised intracranial pressure. Although a half- or one-a-second focus is generally thought to be associated with a severe infiltrating tumour, it can just as readily be produced by a benign tumour, or even with localized lesions due to active cortical atrophy, encephalitis or cerebral thrombosis, whereas conversely a three- to four-asecond focal abnormality may be associated with a very malignant tumour. Very frequently five- to seven-a-second waves appear, so-called theta waves, and these generally indicate the edge of a deeply placed tumour. They have no further reliable significance.

If in the more difficult cases serial E.E.G.s are performed, light will be thrown on the nature of the tumour. Thus a mild abnormality, rapidly worsening, would suggest a malignant tumoup whereas a mild abnormality which did not alte appreciably over months of observation would point to the lesion being relatively benign. It is found that different types of cortical tumour ar $\overrightarrow{\bar{E}}$ more likely to produce a slow wave focus that others, so that different results in E.E.G. diagnosis may be expected. The commoner groups wit any features which may be expected are as follow

I. Astrocytoma, types I and 2

By exhibiting a slow wave focus in the E.E.G. $\vec{P}$ this type of tumour should be localized or lateral ized to the correct hemisphere in 88 per cent. of cases (Figs. 2 and 3 ). The slow wave abnormality is usually seen constantly, but in about 20 peE cent. of the cases it may only be seen occasionallyto If an history of epilepsy is combined with radio logical evidence of brain displacement, as seen by lateral shift of the septum pellucidum, then focal E.E.G. abnormality will invariably occuew This may be of value in differential diagnosis. IQ some cases there may be no slow waves indicative of brain damage over the area involved by the tumour, but epileptic outbursts may be recordee on the affected hemisphere and arise from that part of the cortex directly overlying the astrocytogis

A man of 37 gave an eight-month history. of epileptic fits. Except that his memory had been poor for three months there were, otherwise,

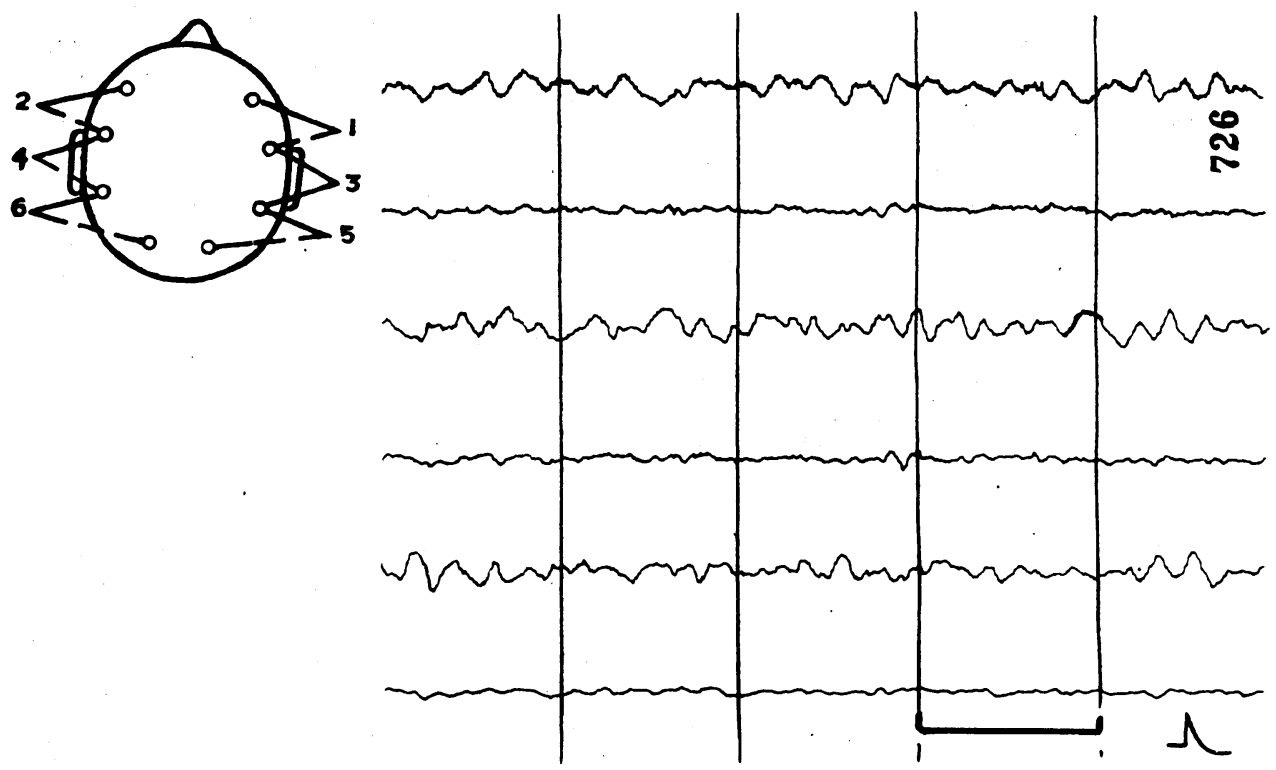

Fig. 3.-Right fronto-temporal astrocytoma extending to the right optic thalamus. Symptoms for 30 months. Ventriculogram showed shift of the septum pellucidum $1.5 \mathrm{~cm}$. towards the left side. High voltage one- to five-a-second waves seen on the right side, whilst there is marked suppression of all frequencies on the left side. 
abnormal signs or symptoms. An E.E.G. showed no slow wave focal abnormality but outbursts of three- to four-a-second waves arose in the right frontal region and spread over the right hemisphere: A ventriculogram showed a right anterior frontal expanding process, and at operation a very cellular astrocytoma measuring 6 by $5 \mathrm{~cm}$. was removed from the right frontal lobe. Two months later the E.E.G. was quite normal and the patient free from symptoms.

\section{Astrocytoma, types 3 and 4 (malignant glioma)}

A similar E.E.G. diagnosis rate is expected with the malignant gliomata as with the benign astrocytomata, but the radiological diagnosis may not so readily be achieved because in many of the cases the tumour is infiltrating rather than displacing the substance of the brain. In about onefifth of the cases X-ray findings may not be contributory in diagnosis, but in these very cases a severe E.E.G. focus will be seen as in the following case, where ventriculography, arteriography and even direct inspection refuted the correct diagnosis :

This was a woman of 67 who had been in good health until four months previously, when she had noted a sensation of numbness in her left hand. Two months later she had a focal fit involving the left hand and the left side of her face. On examination she had a left facial weakness and a left hemiplegia, involving both motor and sensory modalities, which affected the arm more than the leg and was mainly sensory. A persistent right parietal E.E.G. focal abnormality was recorded and because of it, and in spite of both a negative ventriculogram and arteriogram, a crani-otomy was performed. No tumour was found. During the next few weeks her clinical condition worsened and the E.E.G. appearances were seen to deteriorate. When she died, six months after her first symptom, a large infiltrating malignant glioma was exposed at the site where the original E.E.G. record had placed it.

It happens, not infrequently, that a severe focal abnormality is exposed in the routine E.E.G. of the out-patient who presents with symptoms of epilepsy of late onset. The electroencephalogram should not be used as an out-patient filter in the way that mass radiography is used in the control of pulmonary tuberculosis, but when, as the result of study of the record, severe focal brain damage is reported in an out-patient, the case should be admitted to a neurological department for further investigation.

A man of 52 was referred as an out-patient for an E.E.G. He had had an epileptic fit two months previously after which mild right hemiplegic signs were noted but which later improved. An E.E.G. showed a severe clear-cut left temporal lobe abnormality where high voltage two-a-second waves arose. As a result of the F.E.G. report a ventriculogram and exploratory operation were performed to disclose an inoperable malignant glioma. This had arisen from the left caudate nucleus.

\section{Oligodendroglioma}

This type of slow growing neoplasm gives rise to focal slow wave abnormalities, and accurate E.E.G. localization can usually be expected.

\section{Medulloblastoma}

No direct diagnostic help can be expected from E.E.G. study in cases of cerebellar medulloblastoma. Following successful surgical and deep $\mathrm{X}$-ray treatment the generalized high pressure waves are seen to make way for the more normal frequencies. When the inevitable and terminal worsening of the condition sets in the deterioration in the E.E.G. records will be apparent at a very early stage. Deep X-ray therapy to the tumour in the posterior fossa may later cause suppression of the alpha rhythm on the side which has been irradiated.

\section{Pituary Adenoma}

The extrasellar extensions may rise as much as 2 or $3 \mathrm{~cm}$. above the diaphragm and even, in severe cases, obliterate the foramina of Munro, when symptoms due to raised intraventricular pressure will ensue. If, however, there is no change in pressure the largest tumours will but rarely cause alterations in the E.E.G. Five- to seven-a-second waves, occurring in runs, were described in the literature with these tumours, but they seem now to occur in less than 20 per cent. of the cases and they are certainly not diagnostic. Bilateral synchrony of five- to six-a-second waves is more suggestive of a deep mid-line lesion than when runs are recorded, but even so a large proportion of the very gross adenomata may have normal E.E.G. records.

\section{Meningioma}

As a result of clinical, electroencephalographic and pathological findings, it is possible to subdivide the meningiomata into three groups. In the first group, which is associated with a normal E.E.G., the tumour is slow growing, hard, relatively easy to remove and derives its main blood supply from the dura mater. There may be some suppression of the cortical potentials if the tumour is superficial.

A man of $4 \mathrm{I}$ was admitted for investigation with and eight-month history of epilepsy. The initial symptom had been a focal fit commencing in the 
right arm. There were no abnormal physical signs. An E.E.G. showed a dominant frequency at nine-a-second, with generalized underlying fast activity at 22-a-second. There was no evidence of any focal abnormality. A ventriculogram the following day showed poor filling of the ventricular system and a shift of the septum pellucidum of $0.5 \mathrm{~cm}$. to the right of the mid-line. The left foramen spinosum was enlarged. An arteriogram showed displacement of the left anterior cerebral artery $0.5 \mathrm{~cm}$. to the right of the mid-line, and a phlebogram showed evidence of abnormal veins in the left frontal region. At operation a typical meningioma, 7 by $6 \mathrm{~cm}$., was removed from the left fronto-paretal region. It was not unduly vascular and very little trauma was caused at operation to the surrounding tissues. Microscopy showed it to be a rather cellular meningioma. This was a very slow growing parietal meningioma which had not involved the surrounding brain substance and which was associated with an almost normal E.E.G.

The second group in which there is a focal E.E.G. abnormality consist of tumours which may be of the same size as those seen in the first group and have a similar microscopic appearance, but which at operation are found to be extremely vascular and to derive their blood supply from both the meningeal and cerebral vessels. The site of the tumour does not necessarily coincide with the E.E.G. focus.

A woman of 50 had a focal epileptic attack i her left hand seven years previously, following $\underset{\mathbf{a}}{\mathrm{a}}$ mild head injury. For the next three years she was treated for a menopausal "nervous break down,' even though she was suffering from ref current fits in her left hand. During the yeat before admission she had developed recurrent; headaches, deterioration of her eyesight an alteration of personality. On examination she wå mildly demented, had a left facial weakness, and the tendon jerks were increased in the left armb An E.E.G. showed a diffuse abnormality in the right temporal region where medium voltage two-a-second waves occurred in short runs. ventriculogram showed a shift of the septur pellucidum of $2 \mathrm{~cm}$. due to an anterior pariet lesion. At operation an extremely vascular supere ficial meningioma, 5 by $5 \mathrm{~cm}$., lying just above and behind the Sylvian point was removed. It was if $\mathrm{cm}$. thick. Microscopically it was a meningiomeg with widely dilated lymph and blood spaces. This was a very vascular parietal meningioma with $\underline{\underline{\mathrm{a}}}$ slow wave focus adjacent to the superficial site of the tumour.

The meningiomata-en-plaque make up the third group, in which a slow wave focus is found instead of the general suppression which at

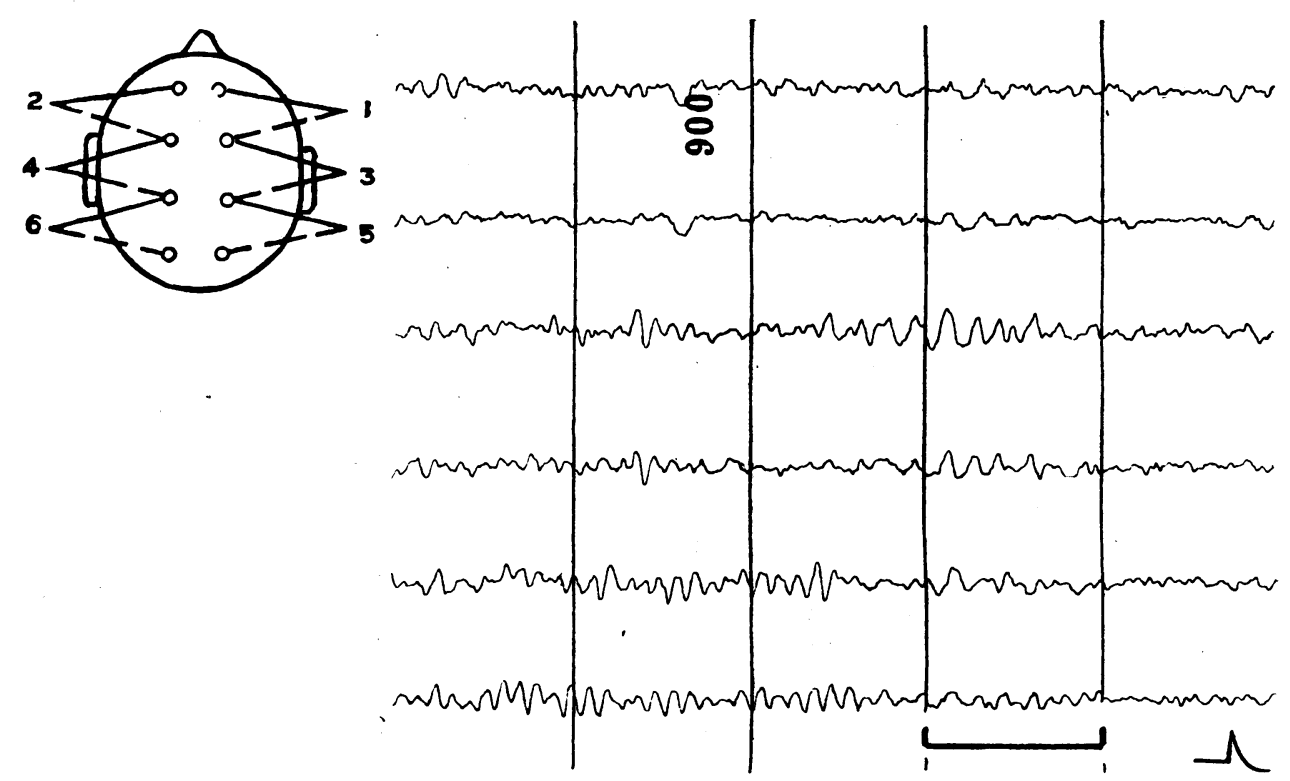

Fig. 5.-Recurrent haemangioblastoma. Symptoms of postural headache and unsteadiness when walking. A ventriculogram was considered to rule out a recurrence of a cerebellar haemangioblastoma, and the ventricular pressure was normal. The E.E.G., however, showed a general abnormality; the alpharhythm of ro-a-second is disturbed in all leads by runs of 5 -asecond waves. 


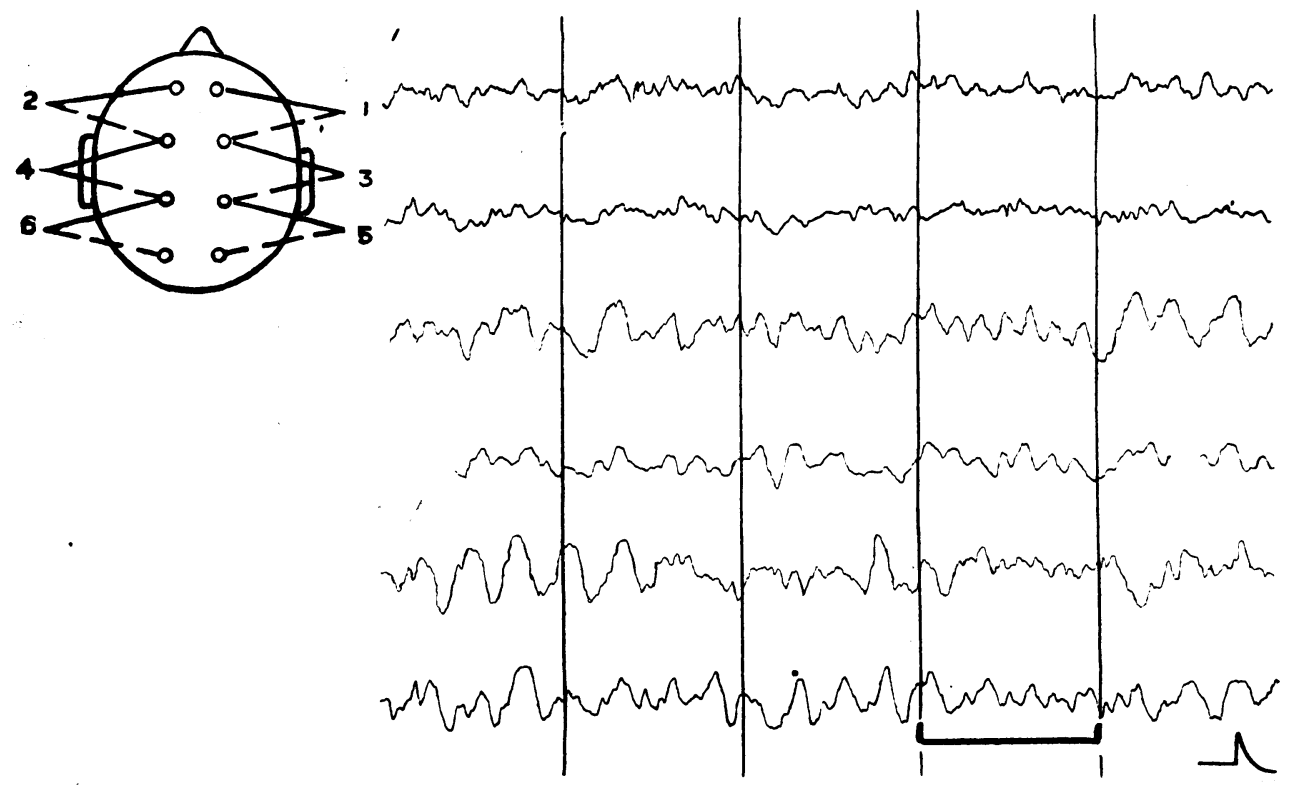

Fig. 6.-Five weeks later he had developed symptoms of raised intracranial pressure with papilloedema. Note the change in the character of the records, high voltage $\mathbf{5}$-a-second waves now occurring in all leads. A week later an haemangioblastoma $3 \mathrm{~cm}$ in diameter was removed. Ten days later the E.E.G. was quite normal.

sight would have appeared probable. The reason for this unexpected finding is that this type of meningioma is not, in fact, a flat innocent plaque but is a vascular and infiltrating mass firmly joined with the brain substance.

The slow distortion of the brain by a meningioma frequently results in the appearance of pathological fast activity over both hemispheres. These fast, beta waves, are generally at 22 -a-second and are seen more commonly with the meningiomata than with any other type of cerebral tumour. In common with the other cerebral tumours the parasaggital lesions are exceedingly difficult to localize, and indeed a normal record may be obtained with very vascular meningiomata occurring at this site.

\section{Haemangioblastoma}

These tumours cannot be localized, nor even lateralized, by E.E.G. methods owing to their situation in the posterior fossa. Slowing of the alpha rhythm may occur as the earliest physical sign of altered cortical metabolism in the earliest stages of the development of raised intracranial pressure and may be present at a time when the intraventricular pressure, as measured at ventriculography, may be considered normal (Figs. 5 and 6). After successful removal of an haemangioblastoma the alpha rhythm should return to its normal frequency and become stable.

\section{Cholesteatoma}

Although these large avascular mid-line frontal tumours are usually associated with symptoms of epilepsy and may slowly displace the frontal lobes, the electroencephalogram is generally found to be quite normal.

\section{Craniopharyngioma}

Unlike the chromophobe adenomata these midline suprasellar tumours give rise to well-marked slow wave abnormalities, probably because of lateral extensions of the cyst. The E.E.G. will show which frontal lobe is the more affected and will assist the surgeon to determine through which side he should approach the tumour at operation.

\section{Metastatic Tumours}

Generally the appearances are similar to those encountered with the gliomata, but the records may contain more than one focal abnormality, these indicating the presence of multiple lesions.

\section{Tuberculoma}

The E.E.G. records are similar to the avascular meningiomata, which they may also resemble macroscopically, and they are generally quite normal (Fig. 4). This is in direct contrast with the severe focal abnormalities produced by localized tuberculous arteritis over the cortex. The E.E.G. can be used for this type of differential 


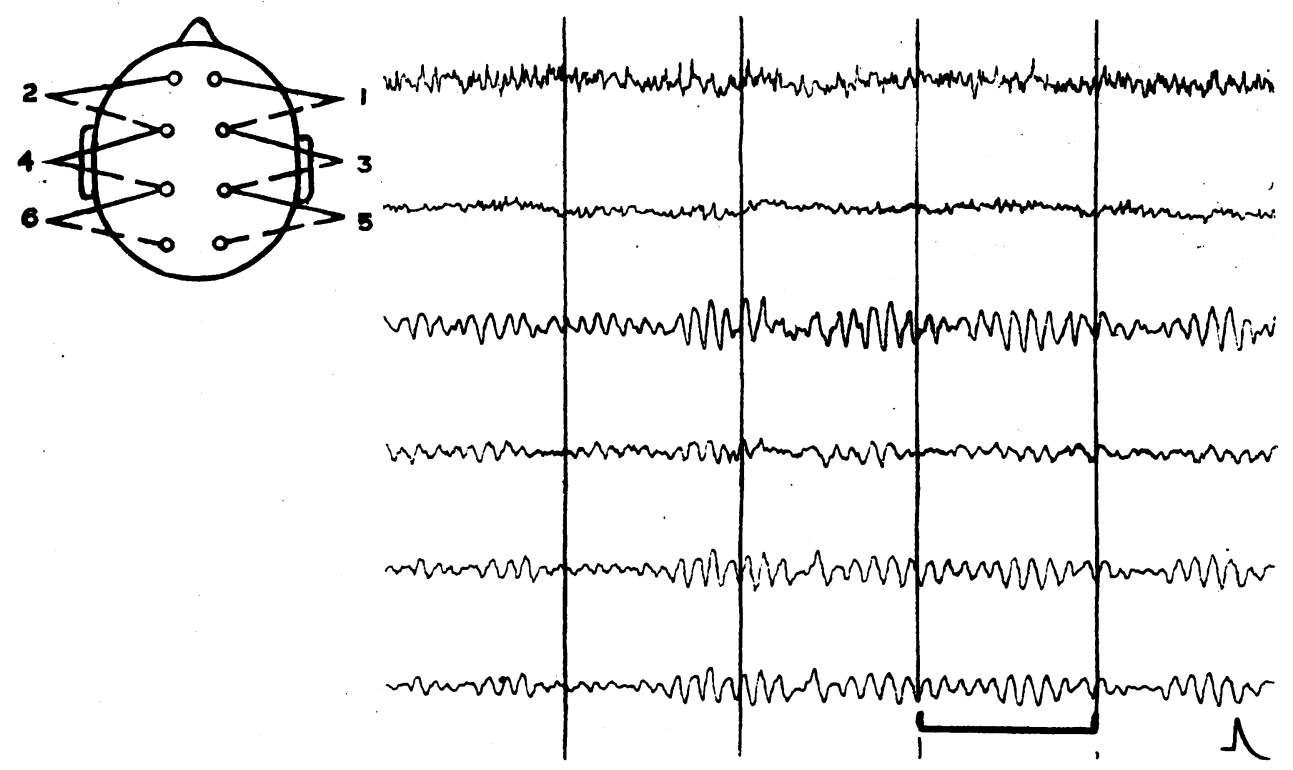

FIG 4.--Right parietal parasaggital tuberculoma. A fairly stable alpha rhythm of ro-a-second is seen over both hemispheres. The increase of the voltage on the right side is due to the presence of a burr-hole in the right parietal area.

diagnosis in those tuberculous patients who develop signs of cerebral disease.

\section{Subdural Haematoma}

Although close clinical resemblance exists between an intracerebral tumour and a chronic subdural haematoma, the E.E.G. is a guide in differentiating between the two conditions. Even with distortion of the brain, as seen by gross displacement of the septum pellucidum, records from an anterior subdural haematoma will be quite normal, whereas in the posteriorly placed lesion there will be suppression of the dominant frequency on the affected side.

A woman of 58 had an 18 -months' history of mental changes. For a year she had been increasingly irritable, and for six months had had difficulty with her speech. There was no history of head injury. On examination she was retarded but there was no evidence of abnormality in her nervous system. An E.E.G. was quite normal. A ventriculogram showed a large left posterior frontal expanding lesion with a shift of the septum pellucidum of $1.5 \mathrm{~cm}$. to the right. At operation a tense bluish dura was incised to reveal a longstanding subdural haematoma containing thick, bright yellow fluid. The membranes were removed to reveal convolutions which though flattened were otherwise normal. She made a rapid and complete recovery following operation.
The presence of a slow wave focus in a provenํํำ case indicates that there has been recent damage to the underlying cerebral cortex which has nof as yet, resolved.

A district nurse of $5 \mathrm{I}$ had a short history of mental deterioration and a right hemiparesis. An $\frac{0}{\circ}$ E.E.G. showed suppression of the alpha rhythm $\cong$ in the left occipital region but there was also a $\overrightarrow{0}$ mild diffuse abnormality in the left temporal lobe 3 consisting of low voltage one- to three-a-second waves. At operation $100 \mathrm{cc}$. of dirty brown fluid? escaped from a left posterior parietal burrhole. It was considered that the haematoma was about four weeks old. She made a rapid recovery and two weeks later the E.E.G. was quite normal; showing that resolution of the damaged brain was complete.

\section{Cerebral Abscess}

An acute untreated cerebral abscess gives rise to an unusually severe and very localized slow $N$ wave focus consisting of high voltage half- to one- $N$ a-second waves which completely exclude all other activity. Recordings from other parts of $\omega$ the hemispheres may be only slightly abnormal. If treatment has been commenced with antibiotics the changes are less specific.

\section{Clinical Application}

Electroencephalography can be used in several ways in cases suffering from cerebral tumour and, $\frac{?}{\mathbb{Q}}$ 
broadly, these can be divided into three groups. First in the selection of cerebral tumours; this particularly applies to out-patient cases in whom diagnosis of cerebral tumour is suspected; secondly in the differentiation of the cases whose investigation for cerebral tumour have already commenced; and thirdly in the control of the proven cases of cerebral tumour.

\section{Selection}

The occurrence of epileptic fits in an out-patient is the most frequent cause for reference for electroencephalographic examination. If the E.E.G. shows a very severe slow wave focus with superadded paroxysms of epileptic activity, then that patient must be suffering from symptomatic epilepsy. Conversely, if short paroxysms of true spike and wave activity, or bursts of generalized fast activity, disturb and otherwise normal record it is more probable that the patient is suffering from idiopathic epilepsy. There will, of course, be many intermediate types in which the E.E.G. appearances are not specific and on which no firm differential diagnosis can be made. The idiopathic epileptic patient will be treated medically, but the patient suffering from symptomatic epilepsy will require further study and investigation. In many cases the optimum time for commencing these further investigations can be found from a study of the E.E.G. records. The appearance of a severe focal abnormality in the E.E.G. of a patient with symptomatic epilepsy may precede by months, or even years, the onset of physical signs, or localizing symptoms, and during this time, if the clinical picture permits, it will be safe to keep the patient under careful medical observation, at first repeating the records every two weeks and later, if there has been no clinical deterioration, every two months.

If the severe focal abnormality lessens and the improvement continues over several weeks, it is unlikely that the patient is suffering from an infiltrating tumour but more probable that he has an area of active cortical disease such as results upon localized vascular insult, with cortical atrophy, or following trauma or infection. On the other hand, steady worsening of the records is produced by a progressive lesion and is almost certainly due to a cerebral tumour. If steady deterioration is seen the patient should be referred for a neurosurgical opinion. The occurrence of generalized high pressure waves in the record, which may be present without there being any papilloedema, demands that air study be carried out by ventriculography rather than by encephalography, which would be unsafe.

\section{Differentiation}

Examination of the features of the various. cerebral tumours which can be expected in any case has already been described and in this way the E.E.G. may be used in many cases as a differential diagnostic aid. It is essential, however, always to add the E.E.G. results to the clinical and radiological findings, thus, in a case which by ventriculography is shown to have a large hemisphere lesion and which has a normal record, the likelihood of the tumour being a glioma is remote and it will almost certainly be an avascular type of condition. Similarly, the patient who shows but indefinite radiological signs and yet has a rapidly worsening E.E.G. most probably has an infiltrating and highly malignant tumour.

It is generally valueless, in differential diagnosis, to depend on only one record and serial recordings will be found to throw considerable light on the nature of the lesion. It is, of course, quite easy to carry out serial recordings in special neurological centres, the method not distressing the patient, being quite painless and devoid of risk. By study of the various appearances which have been detailed above, it will be seen that if a normal E.E.G. is obtained in a case of cerebral tumour, the method cannot be considered to have failed, but it has in fact thrown light on the nature of the tumour. This particularly applies in the meningiomata. The presence of more than one focal abnormality suggests that the lesions are metastatic, though if these are observed in both frontal areas it is more likely that there has been a spread of a primary frontal tumour into the corpus callosum. The practice of so-called blind analysis is quite worthless and in every case as many details as possible should be given to the physician who is interpreting the record so that the maximum value of the method can be obtained.

\section{Control}

After surgical or radiological treatment has been given to any case of cerebral tumour, the records will be seen to improve in a dramatic way in a successful case; persistence of a severe focal 'abnormality, however, is of poor prognosis and heralds the return of the patient's symptoms. The occurrence of post-operative epilepsy may be anticipated by the appearance in the records of an increasing amount of paroxysmal epileptic activity and the appearance of these larval attacks may precede by years the development of clinical-fits. The first sign of return of symptoms due to a recurrence of the tumour will either be focal or, in the case of posterior fossa tumours, will be generalized when slowing of the alpha rhythm is the earliest abnormality to be seen. Complete absence of the cortical potentials in any one particular area following treatment indicates that absolute cell destruction has taken place there, and 
that no resolution is possible. Any persistent symptoms connected with the affected area can be expected to be permanent.

\section{Conclusions}

The electroencephalogram must be regarded merely as an ancillary method of diagnosis when it is used in cases of cerebral tumour and, just as the results obtained from other forms of laboratory study are supplementary to the clinical findings, so the electroencephalographic reports in each patient must be blended by the neurologist with the pathological and radiological findings to form a broad background to the clinical scene.
The electroencephalogram should not be em ployed to demarcate the anatomical limits of a气 cerebral tumour, since this can be achieved so 3 accurately in the majority of cases by the various $\stackrel{\mathbb{Q}}{\Omega}$ radiological methods, but rather should it be used. as a sensitive indicator of the changes in cortical $\vec{F}$ physiology which may be brought about in states? of cerebral pathology. In this way the electro- $\frac{C}{-}$ encephalogram will be found to represent a new dimension in the widening scheme of intracranial diagnosis.

The electroencephalogram can play an importantis part in the observation and control of proven cases $\vec{\circ}$ of cerebral tumour.

\section{BIBLIOGRAPHY}

ADRIAN, E. D. (1935), Proc. Roy. Soc. Med., 29, 197.

BASSETT, R. C., and BAGCHI, B. K. (1947), Univ. Hosp. Bull. Ann. Arbor., 13, II.

BAUDOUIN, A., PUECH, P., FISCHGOLD, H., and LERIOUEKOECHLIN, A. (1946), Sem. H p., Paris, 26, 1226.

BERGER, H. (193I), Arch. f. Psychiat., 94, 16.

BERGER, H. (1933), Ibid., IO0, $30 \mathrm{I}$.

BUCHTHAL, F., and BUSCH, E. (1947), Acta. Psych. Neurol., 22, 9.

COBB, W. A. (1944), F. Neurol. Neurosurg. Psychiat., 7 .96.

COBB, W. A. (1950), Chap. 9, ' Electroencephalography,' edited by Hill, J. D. M., and Parr, G.; Macdonald and Co.

GIBBS, F. A., MONRO, D., and WEGNER, W. R. (1941), New Eng. $\dot{F}$. Med., 225, 279.

\section{'CONCERNING ANAESTHESIA'}

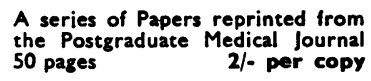

SPINAL ANALGESIA

By William W. Mushin, M.A., M.B., B.A., F.F.A., R.C.S. INHALATION ANAESTHESIA

By John Gillies, C.V.O., M.C., M.B., Ch.B., F.R.C.S.E., D.A.

\section{A SYSTEM OF ANAESTHESIA USING CHEST SURGERY}

By T. Cecil Gray, M.D., D.A., F.F.A., R.C.S.

ANALGESIA IN OBSTETRICS

By P. J. Helliwell, M.B., Ch.B., D.A., and A. Michael Hutton, M.R.C.S., L.R.C.P., D.A.

GENERAL ANAESTHESIA FOR DENTAL SURGERY

By W. S. McConnell, M.B., B.S., D.A.

REGIONAL ANAESTHESIA

By Alan Hunt, D.M., M.Ch., F.R.C.S.

POST-OPERATIVE CARE

By John Beard, M.D., D.A.

Published by

THE

FELLOWSHIP of postgraduate medicine 60 Portland Place, W.I
HINES, C. G., TENNY, L. H., and HUGHES, J. (1944), Navin Med. Bull.'Wash., 42, 10.

HOEFER P. F. A, SCHLESINGER, E. B., and PENNES, H. H. (1946), Trans. Amer. Neurol. Ass., 52.

KERSHMAN, J., CONDE, A., and GIBSON, W. C. (1949) Arch. Neurol. Psychiat., 62, 255.

PARSONS-SMITH, B. G. (1949), 'M.D. Thesis,' Cambridgê University.

SCHLESINGER, B., and STRAUSS, H. (1947), f. Mt. Sind? Hosp., 14, 47.

WALTER, W. G. (1936), Lancet, ii, 305.

YEAGER, C. L., BALDES, E. J., CRAIG, W. MCK., and WOLT MAN, H. W. (1940), Proc. Mayo Clin., 15, 147

YEAGER, C. L., and LUSE, S. S. (1945), Arch. Neurol. Psychiat. 54, 197 .

CEREBRALTUMOURS

Reprinted from the Postgraduate Medical Journal PRICE :

2/9

POST FREE

EDITORIAL: SIR VICTOR HORSLEY

THE PATHOLOGY OF INTRACRANIAL TUMOURS

Dorothy S. Russell, M.D. (Lond.), Sc.D. (Camb.). F.R.C.P. (Lond.)

VIRAL INFECTIONS OF THE HUMAN NERVOUS SYSTEM

Albert B. Sabin, M.D.

TUMOURS OF THE FRONTAL . LOBE

Geoffrey Jefferson, M.S., F.R.C.S., F.R.C.P., F.R.S.

PITUITARY, PINEAL AND THIRD VENTRICLE TUMOURS Joe Pennybacker, M.D., F.R.C.5.

CEREBRAL aNGIOGRAPHY

J. W. D. Bull, M.A., M.D., M.R.C.P., D.M.R.

THE ORTHOPAEDIC REHABILITATION OF A PATIFNT

AFTER EXCISION OF A CEREBRAL TUMOUR

Wylie McKissock, O.B.E., M.S., and K. I. Nissen, F.R.C.S.

SPASTIC PARAPLECIA IN MIDDLE AGE

Colin Edwards, M.R.C.P.

Published by

THE FELLOWSHIP OP

postgraduate mediciNe

60, Portland Place, London, W.I 